University of Wollongong

Research Online

Faculty of Engineering and Information

Faculty of Engineering and Information

Sciences - Papers: Part A

Sciences

$1-1-2014$

\title{
Control issues of distribution system automation in smart grids
}

Kashem M. Muttaqi

University of Wollongong, kashem@uow.edu.au

Ali Esmaeel Nezhad

Islamic Azad University

Jamshid Aghaei

Shiraz University of Technology, jamshid@uow.edu.au

Velappa Ganapathy

University of Malaya

Follow this and additional works at: https://ro.uow.edu.au/eispapers

Part of the Engineering Commons, and the Science and Technology Studies Commons

Research Online is the open access institutional repository for the University of Wollongong. For further information contact the UOW Library: research-pubs@uow.edu.au 


\title{
Control issues of distribution system automation in smart grids
}

\begin{abstract}
In recent years, the world has been exposed to many developments in different areas esp. computer technology, resulting in computers with high power of processing to be built. Among these devices, intelligent electronic devices (IEDs) have the capability to process considerable volume of data at high speed. Since, real-time data processing is vital in distribution networks as the largest users during their operation, IEDs would be applicable in such systems. In addition to IEDS, communication systems have improved during recent decades, providing the desired conditions for a concept known as distribution system automation (DSA) which has been discussed in this paper. Furthermore, the application of distributed generation (DG) in the context of DSA is addressed. Then, different control schemes have been investigated for DG sources while power quality $(P Q)$ issues with DSA in microgrids are proposed in this paper. Moreover, the global automation standard has been presented and a combined strategy is suggested for demand-side management (DSM).
\end{abstract}

Keywords

control, smart, issues, grids, distribution, system, automation

Disciplines

Engineering | Science and Technology Studies

\section{Publication Details}

K. M. Muttaqi, A. Esmaeel. Nezhad, J. Aghaei \& V. Ganapathy, "Control issues of distribution system automation in smart grids," Renewable and Sustainable Energy Reviews, vol. 37, pp. 386-396, 2014.

This journal article is available at Research Online: https://ro.uow.edu.au/eispapers/2907 


\title{
Control Issues of Distribution System Automation in Smart Grids
}

\section{Kashem M. Muttaqi ${ }^{1}$, Ali Esmaeel Nezhad ${ }^{2}$, Jamshid Aghaei ${ }^{1}$, and, Velappa Ganapathy ${ }^{3}$}

\author{
${ }^{1}$ Australian Power Quality and Reliability Centre, School of Electrical, Computer and \\ Telecommunications Engineering, University of Wollongong, New South Wales, Australia \\ ${ }^{2}$ Department of Electrical Engineering, Najafabad Branch, Islamic Azad University, Najafabad, \\ Iran \\ ${ }^{3}$ Department of Electrical Engineering, Faculty of Engineering, University of Malaya, Kuala- \\ Lumpur, Malaysia
}

Corresponding Author: Jamshid Aghaei, University of Wollongong | School of Electrical, Computer and Telecommunications Engineering | Building 35| Northfields Avenue | Wollongong, NSW, 2522 | Ph: +612 $4221 \quad 3413$ | Fax: +612 42213236 | Email: (Jamshid@uow.edu.au)

\begin{abstract}
In recent years, the world has been exposed to many developments in different areas esp. computer technology, resulting in computers with high power of processing to be built. Among these devices, Intelligent Electronic Devices (IEDs) have the capability to process considerable volume of data at high speed. Since, real-time data processing is vital in distribution networks as the largest users during their operation, IEDs would be applicable in such systems. In addition to IEDS, communication systems have improved during recent decades, providing the desired conditions for a concept known as Distribution System Automation (DSA) which has been discussed in this paper. Furthermore, the application of Distributed Generation (DG) in the context of DSA is addressed. Then, different control schemes have been investigated for DG sources while Power Quality (PQ) issues with DSA in microgrids are proposed in this paper.
\end{abstract}


Moreover, the global automation standard has been presented and a combined strategy is suggested for Demand-Side Management (DSM).

Keywords: Distribution System Automation, Power Quality, Distributed Generation, DemandSide Management, Smart Grid

\section{Introduction}

IEDs have been developed drastically these years, mainly because of enhancement in processing power of computers. It is worth-mentioning that IEDS are digital relays having the ability to monitor the real-time system values on the local part of the electric network while such devices can provide the electrical distribution system with extra functionality. Besides monitoring the standard values, such as volts, amps, MW and MVAr, these devices are able to get the data on fault event logs, metering values and oscillography (Voltage, current, power waveforms and harmonics). Thus, it would be useful to replace any faulty protection or monitoring relay with IEDs to move towards DSA. The development of IEDs has led to processing a remarkable data quantity at high speed. One of the industries involving real-time data processing is electric distribution network during its operation. Consequently, a new concept has been introduced to the power industry as DSA which is highly based on recent improvements in intelligent devices and communication protocols. DSA has been defined by IEEE as online remotely monitoring, coordinating and operating distribution network by an electric utility [1]. The need for true automation of distribution network was first realized due to conventional control methods that are all out-of-date [2]. The main factors beyond DSA development would lie in deficits of the conventional system, new competitive market [2] and legislation [3]. In recent years, Australian distribution companies and specifically, New South Wales (NSW) distribution system companies have invested a remarkable cost to enhance the reliability and also to care for extra load growth occurring near the main load centres. Besides the investment programs, the electric distribution industry is moving towards automation of the system as well as ways of implementing DSA.

Many new devices and systems must be installed in the distribution network to thoroughly implement DSA that cause severe challenges for electric utilities in the case of moving from the 
old system towards the new automated one. In order to maximize the benefit, utilities have already started installing new equipment, e.g. new devices, communication system as well as modern softwares, and hardwares [4]. The system used to control distribution networks all across the world is known as Supervisory Control And Data Acquisition (SCADA). This controlling structure involves a system operator to employ the available data in decisions of distribution network control [4].

An important item that has been recently added to distribution networks is DG. The concept of DG has been involved with local generation, i.e. energy conversion units that are located near the consumers [5]. DG esp. from renewable technologies can play a significant role to meet future energy demand while it has potential to decrease Greenhouse Gas (GHG) emission and enhance energy security and adding supply to meet rising energy demand, as well [6-9]. In general, a system consisting of DG to a large extent can be taken into consideration as an environmentally friendly alternative to conventional power supply systems [10]. However, installing DG has its own challenges and merits.

Generally, DG is defined as generation connected to the distribution system at lower voltage levels, i.e. $400 \mathrm{~V}$ to $11 \mathrm{KV}$ levels. DG is normally has low capacity but it is able to relieve the distribution system during peak-load periods [11]. Typical distributed energy sources are solar, wind, wave and geothermal generation, etc. Ref. [12] provides a complete review on distributed multi-generation like Combined Heat and power [CHP] systems. Advantages of DG would be in reducing emission as the main merit as well as reducing power loss and increased reliability in power system [13, 14], while comprehensive review on reliability models used to estimate Renewable Energy Sources (RESs) can be found in ref.[15]. Many benefits may be obtained from using DG in distribution networks, such as supplying peak load which is taken into account as a severe challenge in conventional base load generation. The shortfall with conventional base load generation is in the case of providing additional capacity during peak-demand periods, although they usually provide large centralized generation capacity. DG technologies would be appropriate alternatives to serve the peak load on extreme weather like extremely hot or cold weather while providing the distribution system with RESs [11]. 
When DG is employed to smooth the peak load, it forms part of a concept known as DSM. It is worth-mentioning that, DSM refers to technologies, actions and programs on the demand-side of energy metres intending to manage or alleviate energy consumption in order to decrease total system cost or even for other purposes, such as reducing emissions [16], as well as balancing supply and demand [17]. In other words, DSM has been proposed as an effective program to achieve sustainable development $[18,19]$. On the other hand, in spite of all good features of DG, it may bring severe challenges into distribution networks due to the connection of additional generation to the electric grid. Among problems caused by connecting DG to the electric networks, frequency of DG source, synchronizing DG waveform with the grid and also the voltage profile of DG sources with the electric grid are the most important ones [20]. Moreover, the power electronic equipment needed to facilitate this connection would be another concern. The power produced by many types of DG has DC form while power electronic equipment transforms this waveform, so that it can be connected to the distribution network. It is worthmentioning that these equipments themselves cause unwanted disturbances in distribution networks during their operation [11]. However, it is important to control these negative outcomes of DG with its integration into DSA.

\section{DSA control of DG}

The implementation of DSM is mainly based on real-time data as well as the possibility to fast enable DSM procedures, once it is appropriate to achieve the highest benefit of the distribution network. IEDs are responsible to deliver real-time data while this data is transmitted through the installed communication systems. When employing IEDs in electric distribution systems, the inputs obtained from such devices are the monitoring, protection and control links. It is worth to mention that IEDs are synchronised via satellite connection to have real-time analysis. All the data obtained from IEDS are stored by the substation server while the modem is used to concurrently send the data to other IEDs or centralized command for analysis.

Note that, the automated system decides on switching in or out of service to employ DSM. Ripple control methods and broadcasting would be two alternatives to do such actions [21].

\subsection{Control Issues in distribution networks}


The concept of ripple control and its technology is well-known to distribution networks. In this regard, off-peak hot water system is considered as a ripple control system used by utilities to control electrical hot water systems for residential applications. This action is performed using a high frequency signal, usually injected at the zone substation level into the voltage waveform that is detected by a relay having the responsibility to switch the hot water system on or off which efficiently manages the heating load during maximum demand periods [22]. The aforementioned system is instigated on the time basis when power demand is at minimum. Hence, switching extra loads would cause a little stress on distribution network. On the contrary, ripple control system is used by DSA in order to switch in or out DG sources during peak-load periods while it works on the basis of demand readings done by IEDs. Since the time of maximum demand is not known for each day and each section of the network, a ripple control system which is initialized only by time may not be suitable [22]. The ripple control system employed in the proposed DSM control system is initialized through collecting and analysing the real-time data. According to this data, if distribution network is overloaded, certain measures would be required to reduce system stress. However, in order to implement the desired DG source switching, DSA calculates the optimal solution for DG source input and initializes the ripple control system. It is noted that implementing DG sources switching in and out of service may cause several problems in the electric grid. The main concern relates to the delay in the signal propagation. It is essential no delay occurs when a fast response is needed to protect the distribution network equipment as well as to guarantee the service continuity. In spite of this problem with ripple control system, still some major benefits are obtained through employing this system, such as security of control signals and low set up cost. The most remarkable advantage of ripple control system over others is control signal security [22].

\subsection{Broadcast control of DG sources for DSM}

Using broadcast signal has been suggested to enhance the response time of a control system reducing the overloaded conditions in the distribution networks. Broadcast control can be employed in a same way as Power Line Carrier (PLC) with a difference on the type of communication system, i.e. signal is propagated through a broadcast technology, such as a 
wireless network, Next Generation network or radio signal that all of them are faster at signalling DG control but they have security issues with signal.

\subsection{Multi-Agent control system for DSA and DG}

There are many aspects to be considered when attempt to control DG supplies embedded in the distribution system. Coordination of the generation which achieves a global goal whilst also achieving local goals is a fine balance complicating even more as each DG source is connected. A key feature is that, even the most complex DG-embedded distribution grids use a multi-agent approach in conjunction with IEDs to effectively control DG connection/disconnection [23].

Each individual DG source is accompanied by a Local Control Agent (LCA) which monitors and controls the local DG environment. LCA provides additional information to other LCAs and to a Global Control Agent (GCA) overseeing all control inputs and outputs. GCA can also interrupt any LCA that executes a control action which is detrimental to the global system stability. Fig. 1 and Fig. 2 below demonstrate a graphical representation of the multi-agent DG control system [23]. Note that Local Emergency Signal (LES) is transmitted to the rest of the system while GES is Global Emergency Signal that both are reported to coordination and learning section.

\subsubsection{Control process algorithm}

There are several steps in the control algorithm suggested in ref. [23] outlining the operation of the multi-agent DG control system as follows:

1. The ultimate goal of the LCA connected to each DG source is to maintain generation to the local load and also to control local conditions. Only after it has satisfied the local conditions, it also tries to look beyond to the global scenario based on local and global information.

2. For any DG source that fails during operation, it is immediately disconnected from the grid and an emergency signal is sent to all other agents informing the disconnection. A failure of a DG source would be due to any failure in hardware or software associated with the DG source, LCA, or the execution of a control sequence that leads to instability in the distribution system. 
3. Any remaining DG sources will reassess the local and global inputs and determine appropriate action with regard to the new operating conditions.

4. To return the failed DG supply to service, the GCA attempts to find a solution to the optimal control settings through off-line calculation. Once the solution converges to an optimal solution, an attempt to return the DG service is made. This process is known as "self-healing” and it is a key advantage of multi-agent systems.

5. If the GCA does not converge to an optimal solution and the global goal deteriorates, all DG sources are disconnected reverting back to traditional power supply.

6. The GCA then tries to calculate an off-line optimal solution and proceeds to restore all DG sources either automatically or manually depending upon the situation.

The most appealing aspects of this system are self-learning capability, robustness in control and the ability to adapt to add-on functions in future scenarios. The additional expansion of the system to support future control functions augments the attractiveness of the system [23]. Utilities implementing a multi-agent system would install a system with a long shelf life due to the ability to expand the functionality and control, effectively staving off the redundancy curse. These aspects make the system a genuinely suitable consideration for utilities to control DG sources embedded in the distribution grid. The interaction between the local and global agents can be illustrated as Fig. 3.

\subsection{DSA for PQ monitoring and correction under smart grid}

PQ refers to the purity of the voltage and current waveforms within the distribution system. Nominal measurements of the voltage waveform are 400V RMS at distribution level and a frequency of $50 \mathrm{~Hz}$. Most connected loads have low tolerance to PQ events, such as voltage swells and sags, since loads are generally designed to operate within a specific range of voltages. Any excursion into measurements above or below this range will mostly cause the load to fail or equipment protection to be activated [3].

With connection of high-powered electronic equipment to distribution system, it has become an inherent need to monitor the PQ disturbances created by these electronic devices [3]. Moreover, customers connected to the grid move towards using electronic equipment that are increasingly 
more sensitive to power quality disturbances rather than previous technologies. With customers desiring increased PQ and also with connected loads producing more PQ events, monitoring the PQ has become a vital part of the distribution system and DSA provides a possible solution to ensure PQ standards [24].

\subsection{Smart meters}

The wide spread rolling out of Advanced Meter Infrastructure (AMI), also known as smart meters is essential to DSA concept, particularly in the case of improving PQ in distribution systems. AMI has the capability to improve the response time during outage management procedures, monitor and predict harmonic scenarios, take evasive load shedding to avoid PQ disturbances and power outages, monitor current and voltage waveforms, provide DSM initiatives and also it is able to provide consumers with more information on their energy consumption which in turn, could lead to savings on the consumers' energy bill [25]. The smart meter is enabled with two-way communication providing many benefits to both the installed customer and the utility in gaining operational efficiencies [26].

\subsubsection{DSM and smart meters}

The end-user customer can have a major impact on the demand profile of the distribution system while the smart meter makes the customer able to reduce the stress on the distribution system during times of maximum demand [25]. The smart meter facilitates the introduction of an improved billing system which is more in line with actual electricity market conditions.

The Time of Use (ToU) billing system associated with the smart meter allows the customer to defer non-essential energy consumption to times when demand for energy is lower. ToU encourages this deferral of loads to periods with lower demand in the distribution system through time-based tariffs. For periods when energy demand is the highest, the utility charges a higher price reflecting the actual price of energy at that time. The opposite is true when demand is low. The utility charges less for energy used in periods of lower demand to encourage the deferral of non-essential loads to these periods [26].

The smart meter leads to additional functionality useful for the distribution company to provide DSM initiatives. The two-way communication of the smart meter enables the utility to control specific loads in a house. Controlling specific loads can reach beyond the mere switching in or 
out of loads and can be extended to change the thermostat value of an appliance, such as an intelligent air-conditioner in order to lower the energy consumption of the appliance and to reduce load during maximum demand periods which seems necessary [26]. The interaction between smart appliances, the smart meter and the energy providers is illustrated in Fig.4.

The smart meter is the interface between the customer and the energy provider which can provide DSM initiatives using direct control, local load control and distributed control. The utility can remotely switch loads in or out of service via the smart meter and any smart appliances to effect a reduction in demand during periods of energy network stress [23]. Switching via smart meter is achieved through sending a control signal from the utility to the smart meter which then acts on this control signal. This is known as direct control of customer loads and can be implemented through the use of ripple control (PLC) or through broadcast control [22].

Local load control is initiated by the customer. The smart meter central display provides the customer with sufficient information in order to make informed decisions on when and where they use energy within their own premises. Devices, such as timers and demand actuated circuit breakers installed by the customer are examples of local load control where the customer programs these devices to activate them when the device has reached a predetermined value [25]. The final control method is distributed control. Distributed control requires the interaction of both the utility and the customer in order to implement DSM. It is most common between industrial loads and the energy provider to agree a contract based on which, the customer commits that non-essential loads to be switched out of service during periods of high energy demand generally in view of a predetermined compensation value. Note that with the advent of the smart meter and home automation, it may be possible to implement such schemes on a residential level [26]. Here, the customer would have nominated loads which can be shed during periods of high demand with the compensation to effectively help the continuation of service.

The aim of DSM is to involve the customer in the process as much as possible and therefore the order in which, control of loads should be initiated during network stress would be local load control, distributed control and finally as a last resort, direct control. This would ensure that customer satisfaction remains at high levels.

\subsection{Standards and key challenges}


With greater data logging and communication requirements necessary for power system automation, it has become apparent that, certain standards are required to provide compatibility and minimum technological standards across all developed intelligent components. Furthermore, utilities are increasingly installing intelligent relays and protection equipment all with differing communication requirements giving rise to compatibility problems of devices. The development of the IEC 61850 has occurred as a consequence of this apparent need to standardisation and today, it is the published guiding principles for all intelligent devices developed for DSA [27].

The IEC 61850 differs from all other preceding standards when dealing with substation communication and substation to master communication. The uniqueness of the IEC 61850 is due to not only describing the protocols of how the data must be sent but also, how it should be stored in any given intelligent device [27]. This fact is an important shift in the introduction of communication standards between intelligent devices through a power system, since it recognises that there are greater constraints to realise the potential of intelligent devices than just the compatibility of data transmission protocols [28]. Configuration of IEDs can be easily implemented as a consequence of the standardised data formatting within IEDs with a simple upload of a file [27].

\subsection{Key features}

There are multiple features of IEC 61850 that all of which influence the effectiveness of the standard to reduce installation costs and increase compatibility between devices. The followings are key points that are unique to IEC 61850 standard ref.[28].

- All communication protocols, data services and device behaviour are predefined by the standard. The network transmitting any data between intelligent devices that uphold IEC 61850 standard also adopts the strict predefined transfer protocols.

- Unlike previous standards for substation communication and control, which only assigned a location, IEC 61850 gives specific names to all data in the form of strings.

- These data names are standardised across all equipment and have specific industry and meaningful terms. 
- Clients using data from intelligent devices are able to download data types supported by the device without any need for manual configuration, resulting in simplified data extraction.

- The introduction of High Level Services (HLS) adds another dimension to the IEC 61850 standard. The HLS enables additional functionality to be achieved through IEDs on a standardised manner.

- Outlining a Standard Configuration Language (SCL) enables the easy configuration of any intelligent device as well as defining the devices' specific function in the power system network.

Considering the key features of the IEC 61850, it should be mentioned that what impact the above features have when they are standardised. The major benefits are listed below.

\subsection{Major benefits}

The major benefits of IEC 61850 can be listed as follows [28]:

- The precise definition of the requirements for substation automation leads to elimination of procurement ambiguity. Hence, manufacturers of intelligent devices would have a clear definition of what each device needs to be capable of executing.

- By standardising the data transferring and processing, there would be an inevitable reduction in installation costs. This is due to the decrease in configuration time of all network devices. Furthermore, by utilising communication bandwidth, installation of cables reduces and therefore the installation of infrastructure, such as trenches and conduits also reduce that results in saving on installation costs of automated systems.

- The standard reduces the commissioning costs of automated systems, as the configuration process of equipment is simplified and does not need to be undertaken manually. This in turn reduces human errors in configuration.

- The IEC 61850 allows users to supply devices from different manufacturers while knowing that the devices are all compatible. This is due to the device specifications being outlined in the standard with all devices containing the same naming data, naming conventions and transfer protocols. 
- Extending the substation automation system requires less additional costs when systems and devices are all standardised according to IEC 61850. Gathering additional data through existing devices or installing additional devices on the substation system requires minimal interference and minimal inconvenience to existing infrastructure reducing upgrade and extension costs to a large extent.

- The IEC 61850 standard also describes the method of using existing communication infrastructures that many utilities have already used, resulting in a reduction in the integration costs with existing systems. This can also reduce the interfacing costs between old communication techniques and new ones while enabling utilities to avoid new communication channel costs altogether where existing infrastructures exist before.

- IEC 61850 outlines the minimum standards when using existing communications channels and provides detailed provisions for future technological change to ensure a smooth transition between technologies. This is a unique feature of IEC 61850 to not only describe existing standards, but also to lay the foundations for standards and technologies to come.

The above key points are the major features of the IEC 61850 while they are unique to this standard [28]. It is important to recognise that in editing such standards as IEC 61850, there has been an emphasis on the integration of technologies, devices and communications and in no way is designed to reduce the innovation of new products. The standard regulator recognises that a forward program and plan can greatly reduce costs and providing standards for technologies to come is a concerted effort to reduce future costs [27].

DSA is a broad topic involves many different aspects that can be researched and demonstrated. Combined DSM Strategy encompasses many technologies and benefits that only DSA can provide. As the system delves into many issues concerned with DSA, it seems appropriate to focus on these results.

After significantly researching DSM strategies, a proposed combined system of DG source switching, smart meter technologies and Direct Load Control (DLC) is demonstrated and results are discussed. The proposed method deals with the order in which, the time each strategy should be engaged and the time each strategy should come into effect are determined. The order that each strategy is engaged is decided by the level of inconvenience to the customers so that the 
least inconvenient strategy is used first while the most inconvenient is left till last with the hope that the previous strategies have resulted in lower demand required.

Firstly, it must be stated that the strategy presented has endeavoured to account for every possible variable that can affect any one of the separate DSM strategies so that the desired reduction of demand occurs. Particularly, these variables are DG connection failure, variable DG output and the variable nature of load deferral. With these issues in mind, the proposed system is able to reach its ultimate goal, i.e. demand reduction, regardless of the individual contribution of DSM strategies. The overloaded electrical asset does not differ for the strategies, but only benefits from the overall result of demand reduction. The benefit of the strategy is to enhance the interaction of customers and utility to achieve this common goal meeting the needs of both consumer and network.

\section{Combined DSM strategy}

Based on the level of the perceived customer/utility convenience, the order of engaging the DSM strategies is an attempt to connect DG sources, manage the load deferral through smart meters and customer approval and finally reduce demand through DLC.

The aim of utilising DG is first to effectively operate the distribution system as before without

any difference in power delivery or quality noticed by the customer. Indeed, using DG results in the added benefits of improved power factor and receiving end voltage experienced by loads on the downstream side of DG connection. Afterwards, the utility asks customers to curtail nonessential loads during peak times. The utility achieves this through ToU pricing and smart meters, making the customer informed of electricity price at the intended time of use. Further reductions could be gained, if utilities would be able to use AMI and smart appliances to warn consumers of the cost of using an appliance during any high demand time and ask the consumers if they would like to defer their consumption. By asking the customer to lessen their demand, the utility can possibly avoid the last resort option of DLC. DLC states that the utility selects loads to be controlled with no input from the customer in order to reduce the demand on the electrical distribution system. Obviously, this would be the most inconvenient method of reducing demand and likely to cause the customer to become dissatisfied with the utility's service. 


\subsection{Intricacies of combined system}

Systems base their decisions on the previous demand interval to select the best action that needs to be taken if any is required. The most critical demand value is as the nameplate value of the electrical assets, such as a transformer. At a demand greater than $100 \%$ of nameplate value, the utility seeks to connect DG sources embedded within the section of the network concerned. Once all available DG sources have been connected, the utility looks to its customers to reduce their demand on the network through smart meters. Only a fraction of non-essential loads will be shed by the utility's customers and if the demand still exceeds 100 percent nameplate value, then the utility engages DLC to curtail the demand to the value below the nameplate value.

The proposed system goes beyond the reduction of demand during overloaded situations with smart meter and load management strategy having an extra effect in addition to during overloaded periods [25]. The shoulder period of any peak will have load deferrals from the smart meter and load management strategies due to ToU tariffs. At 75 percent of nameplate value, the utility will ask for load deferrals from its customers. As the price of electricity in the shoulder periods is elevated significantly above the base prices, there is still the possibility of load deferrals, albeit the probability that customers defer in the shoulder periods would be much lower than during peak times when the incentive deferring is the highest [25].

Loads that are deferred are loads that will still be connected to grid at some points in time and the system provides incentives to connect these loads during periods of low demand and potentially has the ability to connect these loads through remote load control. The idea behind this action is facilitating the filling of low demand periods with the deferred loads improving asset utilisation. With ToU, the customer also gets benefit, since the price of electricity in this period has been set significantly lower than peak periods [25]. To further smooth the load profile, extra incentives could be introduced by the utility, such as further price reduction if the customer agrees that the utility can remotely switch the load into service. Loads, such as washing machines and dishwashers would be perfect choices for such a scenario. For this additional feature of remote load initiation, it is necessary that the house is compatible with both smart appliances and smart meter. Fig. 5 depicts a flowchart providing a visual explanation of the above-described combined DSM strategy. 


\subsection{Combined DSM strategy algorithm}

The primary concern on combined DSM systems is to limit the network demand on its electrical assets to below the rated value of these assets. Generally, the system is most practical instigated using a critical electrical asset, such as a Zone or sub-transmission substation transformer or significant size. This is because the combined DSM strategy has a profound impact on demand when using the data of a large transformer as the decision variable for the algorithm and the data received is reliable with limited variability from one demand period to the next when compared to smaller transformers [23]. Accordingly, the following equation can be stated to reduce peak demand.

$$
P_{R i}=P_{i}-P_{D G i} \quad \text { if } \quad P_{i}>P_{\text {rated }}
$$

Where $P_{i}$ is the power at time interval $i, P_{\mathrm{DG}}$ is the power delivered by the DG sources and $P_{R}$ denotes the power demand after the DG has been connected. This occurs only when the initial power $P_{i}$ is greater than the rated value of the critical electrical asset $P_{\text {rated }}$. If $P_{R}$ remains greater than $P_{\text {rated }}$, the strategy will insist on load deferral from customers connected to the network.

$$
P_{L M i}=P_{R i}-P_{L D i} \quad \text { if } \quad P_{R i}>P_{\text {rated }}
$$

Where, $P_{L D i}$ denotes the total power of the deferred loads for the time period and $P_{L M i}$ is the power demand after the managed loads have been deferred. Only after the first two strategies have been investigated and $P_{L M i}$ still remains greater than $P_{\text {rated }}$, the utility must employ DLC to further reduce demand. The final equation incorporating load control can be stated as follows:

$$
P_{\text {COMBi }}=P_{L M i}-P_{\text {DLCi }} \quad \text { if } \quad P_{L M i}>P_{\text {rated }}
$$

Where, $P_{\text {сомві }}$ indicates the final power demand after the combined DSM strategy is completed and $P_{L M i}$ is the difference between the power after load management and $P_{D L C i}$ denotes the total controlled power. The total controlled load is always significant enough to reduce the managed load power to below the rated power of the transformer as a consequence of the DLC process.

Secondary to the combined DSM strategy, but of the same importance is to schedule deferred customer loads. The loads are to be scheduled to connect during periods of low demand aiding the utility to fill load profile troughs and as a consequence of ToU tariffs, the customer's power bill will decrease. An estimation of the limiting load factor is needed to reschedule deferred loads 
which can be found using the total deferred load value and the previous days demand profile [25].

Once the limiting demand is found, the utility can begin to schedule deferred loads filling the area between the predicted demand curve and the limiting demand factor.

$$
P_{L F i}=P_{i}+\sum_{i=1}^{P_{L F F}<y} P_{L D i}
$$

Where the power after load filling is denoted by $P_{L F i}$ and $P_{i}$ indicates the actual power for the time period.

The above equation shows that a fraction of the total deferred load is to be added to the actual power until $P_{L F i}$ is approximately equal to the limiting demand $y$, effectively filling the trough in the demand profile.

\subsection{Separate strategies explanation and algorithm}

Central to the above algorithm is how the combined DSM strategy calculates the critical values of, $P_{L D i}$ and $P_{D L C i}$. These values represent the total power able to reduce demand on the system by each separate strategy, namely DG connection, load management and DLC. To understand how these critical values are calculated, each individual strategy must be explained both in isolation from the combined strategy as well as in the context of the combined strategy.

\subsubsection{DG source connection strategy}

When DG sources connect to the distribution grid, there are many variables affecting the input power and connection success. DG input is generally variable; So only a percentage of the rated value of output can be guaranteed. Furthermore, the connection to the grid is not always successful with a local source failing due to synchronisation or voltage problems, so that with a small percentage of these failed connections, a global failure of all DG sources may occur [23]. The DG source connection strategy used in the combined DSM strategy takes these variable factors into consideration during implementation of DSM strategies using DSA.

Firstly, DG connection strategy is based on contractual agreements between DG providers and utility. These contracts state that DG providers will supply energy when the network reaches an overloaded demand value and that the utility commits to buy the generated power from the 
contracted providers. Different DG providers negotiate their own contract with the utility prior to installing DG supply with a set power value guaranteed to connect when a certain demand level is reached. If the DG supplier fails to connect during the contracted period, compensation is sought by the utility because of failing to supply contracted DG. The supplier has the advantage due to supplying energy to the utility at the electricity price set by the market with the utility having an obligation to buy during the contracted period [11]. Because of differing contracts, the connection of various DG supplies is staggered over the range of demand values. Fig. 6 illustrates a flow chart including DG connection strategy.

All attempts should be firstly made to connect DG sources when encountering an overloaded network scenario before engaging any other demand reduction strategy. If a single or global failure occurs during the connection process, the utility has to employ either the next DG source or the next demand reduction strategy. One significant factor which is not taken into consideration in this flow chart is the time of day that generation takes place. This factor would cause problems in the case of some specific energy sources like solar generation which can be considered as the contracted source and therefore, input will diminish as the day continues. DG connections after 3pm are reduced and connections after 6:30pm cannot occur as sunlight decreases.

\subsubsection{Load management}

Load management is relatively straightforward. When the demand reaches a particular value, the utility asks customers to defer their non-essential loads. The process is separated into two initialisation points to meet the different needs of the network during the peak period and the shoulder period of network operation. The varying load deferral between the two periods has been also taken into account, as fewer customers are likely to defer their loads during the shoulder periods rather than during the peak demand periods. In addition, the shoulder load management period is a pre-emptive move to cut demand before the network becomes overloaded and it must be considered as a supporting strategy to the main focus of the combined DSM strategy [25]. The flowchart in Fig. 7 demonstrates this point.

If during the peak demand period that both the DG source connection strategy and the load management strategy fail to result in enough reduction, the utility can look towards more drastic DLC strategy. 


\subsubsection{DLC}

DLC involves a signal propagated through a communication channel in order to control specific loads or groups of loads to reduce demand on the distribution system. The network serviced by the critical asset is broken up into controllable groups with a set demand that can be reduced. By using DLC, the utility can decrease the demand on the critical electrical asset to below the nameplate value regardless of the extent of overloading. For instance, if the air-conditioners on the costumer side can be directly planned, dispatched and controlled, the peak load can be effectively reduced [29]. Note that extremely overloaded assets (approximately $150 \%$ of the rated value) should be upgraded to meet network conditions as DSM strategies are effective only to a certain point before asset replacement is required. The DLC is covered extensively in ref. [30].

\section{Conclusions}

In completing the research on DSA, it must be first stated that the research area covers a broad range of topics that is difficult to cover all aspects completely in any technical depth except virtually writing a book. The DSM strategy comprises DG switching, Load Management and DLC that all of which have been extensively discussed. The combination of these strategies into a single strategy based on customer inconvenience will be a new area of research. The most convenient strategy is employed first while the most inconvenient method is employed as a last resort. The strategy takes into account the needs of both the utility and the customer when operating during peak demand periods. The strategy aims to satisfy the customer's needs before the utilities needs, but does not neglect its overall aim of peak demand reduction. The strategy reduces demand regardless of the technique used, although the strategy aims to reduce the impact on the connected customer.

\section{References}

[1] R. Gupta and R. Varma, "Power distribution automation: present status," Academic open internet journal, vol. 15, 2005. 
[2] C. H. Hauser, D. E. Bakken, and A. Bose, "A failure to communicate: next generation communication requirements, technologies, and architecture for the electric power grid," Power and Energy Magazine, IEEE, vol. 3, pp. 47-55, 2005.

[3] A. P. O. Gustaf, 'The Challenge and Sustainable Energy' Technical Breakfast Briefing Sydney - Areva T\&D Pty, Friday, 24th August, 2007.

[4] Y. Pradeep, S. Khaparde, and R. Kumar, "Intelligent Grid Initiatives in India," in Intelligent Systems Applications to Power Systems, 2007. ISAP 2007. International Conference on, 2007, pp. 1-6.

[5] K. Alanne and A. Saari, "Distributed energy generation and sustainable development," Renewable and Sustainable Energy Reviews, vol. 10, pp. 539-558, 2006.

[6] M. F. Akorede, H. Hizam, and E. Pouresmaeil, "Distributed energy resources and benefits to the environment," Renewable and Sustainable Energy Reviews, vol. 14, pp. 724-734, 2010.

[7] M. B. Amor, P. Lesage, P.-O. Pineau, and R. Samson, "Can distributed generation offer substantial benefits in a Northeastern American context? A case study of small-scale renewable technologies using a life cycle methodology," Renewable and Sustainable Energy Reviews, vol. 14, pp. 2885-2895, 2010.

[8] A. F. Sherwani, J. A. Usmani, and Varun, "Life cycle assessment of solar PV based electricity generation systems: A review," Renewable and Sustainable Energy Reviews, vol. 14, pp. 540-544, 2010.

[9] Varun, I. K. Bhat, and R. Prakash, "LCA of renewable energy for electricity generation systems-A review," Renewable and Sustainable Energy Reviews, vol. 13, pp. 10671073, 2009.

[10] M. Wolsink, "The research agenda on social acceptance of distributed generation in smart grids: Renewable as common pool resources," Renewable and Sustainable Energy Reviews, vol. 16, pp. 822-835, 2012.

[11] J. Dolezal, P. Santarius, J. Tlusty, V. Valouch, and F. Vybiralik, "The effect of dispersed generation on power quality in distribution system," in Quality and Security of Electric Power Delivery Systems, 2003. CIGRE/PES 2003. CIGRE/IEEE PES International Symposium, 2003, pp. 204-207.

[12] G. Chicco and P. Mancarella, "Distributed multi-generation: A comprehensive view," Renewable and Sustainable Energy Reviews, vol. 13, pp. 535-551, 2009.

[13] P. E. Labis, R. G. Visande, R. C. Pallugna, and N. D. Caliao, "The contribution of renewable distributed generation in mitigating carbon dioxide emissions," Renewable and Sustainable Energy Reviews, vol. 15, pp. 4891-4896, 2011.

[14] W.-S. Tan, M. Y. Hassan, M. S. Majid, and H. Abdul Rahman, "Optimal distributed renewable generation planning: A review of different approaches," Renewable and Sustainable Energy Reviews, vol. 18, pp. 626-645, 2013.

[15] C. L. T. Borges, "An overview of reliability models and methods for distribution systems with renewable energy distributed generation," Renewable and Sustainable Energy Reviews, vol. 16, pp. 4008-4015, 2012.

[16] Z. Ming, X. Song, M. Mingjuan, L. Lingyun, C. Min, and W. Yuejin, "Historical review of demand side management in China: Management content, operation mode, results assessment and relative incentives," Renewable and Sustainable Energy Reviews, vol. 25, pp. 470-482, 2013. 
[17] P. Warren, "A review of demand-side management policy in the UK," Renewable and Sustainable Energy Reviews, vol. 29, pp. 941-951, 2014.

[18] H. Ma, L. Oxley, and J. Gibson, "China's energy situation in the new millennium," Renewable and Sustainable Energy Reviews, vol. 13, pp. 1781-1799, 2009.

[19] M. Zeng, C. Li, and L. Zhou, "Progress and prospective on the police system of renewable energy in China," Renewable and Sustainable Energy Reviews, vol. 20, pp. 36-44, 2013.

[20] L. Kojovic, "Impact DG on voltage regulation," in Power Engineering Society Summer Meeting, 2002 IEEE, 2002, pp. 97-102.

[21] A. T. de Almeida and E. L. Vine, "Advanced monitoring technologies for the evaluation of demand-side management programs," Energy, vol. 19, pp. 661-678, 1994.

[22] D. Raisz and A. M. Dan, "Ripple Control as a possible tool for daily load balancing in an open electricity market environment," in Power Tech, 2005 IEEE Russia, 2005, pp. 1-6.

[23] A. Al-Hinai and A. Feliachi, "Application of intelligent control agents in power systems with distributed generators," in Power Systems Conference and Exposition, 2004. IEEE PES, 2004, pp. 1514-1519.

[24] J. D. Bouford and C. A. Warren, "Many states of distribution," Power and Energy Magazine, IEEE, vol. 5, pp. 24-32, 2007.

[25] De Almeida .A and Vine .E, "Advanced Monitoring Technologies for the Evaluation of Demand Side Management Programs," IEEE Transactions on Power Systems, vol. 9, pp. 1691-1697, August 1994.

[26] J. Brogden, "ERA View on Smart Metering and Interoperability," in proceeding of: Smart Metering - Gizmo or Revolutionary Technology, IET seminar on, 2008, pp. 1-19.

[27] T. Sidhu and P. K. Gangadharan, "Control and automation of power system substation using IEC61850 communication," in Control Applications, 2005. CCA 2005. Proceedings of 2005 IEEE Conference on, 2005, pp. 1331-1336.

[28] R. Mackiewicz, "Overview of IEC 61850 and Benefits," in Power Systems Conference and Exposition, 2006. PSCE'06. 2006 IEEE PES, 2006, pp. 623-630.

[29] G. C. Heffner and D. A. Kaufman, "Distribution Substation Load Impacts of Residential Air Conditioner Load Control," Power Apparatus and Systems, IEEE Transactions on, vol. PAS-104, pp. 1602-1608, 1985.

[30] C. Y. Chang, C. J. Wu, C. T. Chang, C. H. Lin, J. L. Yen, T. G. Lu, J. L. Yen, T. G. Lu, W. C. Chang, "Experiences of direct load control using ripple signals in Taiwan power system," IET Conference Proceedings of Advances in Power System Control, Operation and Management, vol. 2, pp. 591-596, Nov. 1997. 